\title{
Inversor resonante para ahorro de energía en un calentador de agua mediante inducción magnética
}

Fecha de recepción : 15 de octubre de 2018 • Fecha de aceptación: 9 de noviembre de 2018 • Fecha de publicación: 7 de enero de 2019

Ing. Galo Fabian Acosta Rivera

Empresa Eléctrica Quito, Ecuador fabiancbr_86@hotmail.com

\section{Resumen}

El calentamiento por inducción magnética se ha implantado con satisfacción en procesos para recocido de piezas cerámicas, endurecimiento superficial, fusión, forjado en caliente, soldadura, entre otros, demostrando así su versatilidad y efectividad. En este artículo se detalla el diseño y la construcción de un prototipo de calefacción para piletas de agua mediante inducción magnética. Su funcionamiento está basado en un inversor cuasi resonante encargado de generar ondas electromagnéticas de alta frecuencia, las cuales inducen corrientes parásitas de alto valor que producen el calentamiento de los elementos ferromagnéticos cercanos a la bobina de inducción. El aporte de esta investigación es el estudio de la energía consumida y el tiempo empleado a diferentes niveles de potencia.

\section{Palabras claves: inducción magnética, ferromagnético, calentamiento por inducción.}

\begin{abstract}
The heating by magnetic induction has been successfully implemented in processes for annealing ceramic pieces, surface hardening, melting, hot forging, welding, among others, thus demonstrating its versatility and effectiveness. In this article the design and construction of a prototype heating for water pools by magnetic induction is detailed. Its operation is based on a quasi-resonant inverter responsible for generating high frequency electromagnetic waves, which induce high value parasitic currents that produce the heating of ferromagnetic elements close to the induction coil. The contribution with this research is the study of the energy consumed and the time spent at different power levels.
\end{abstract}




\section{Introducción}

Los sistemas de inducción magnética son desarrollados bajo el principio de Faraday (1831). En la actualidad estos sistemas tienen aplicación tanto en el ámbito doméstico como en el industrial. Bajo este principio se han confeccionado motores eléctricos, transformadores, generadores, entre otros; sin embargo, su aplicación no es perfecta, ya que por su propia naturaleza se producen pérdidas de energía durante la inducción electromagnética. Estas pérdidas afectan negativamente la funcionalidad y el rendimiento en un sistema eléctrico (López, 2016). Para la realización de este proyecto se propone aprovechar las pérdidas generadas por el fenómeno de inducción antes expuesto al convertirla en energía térmica para el proceso de calentamiento por inducción.

En ese sentido, el procedimiento consiste en construir una bobina con un núcleo de material ferroso, el cual se calentará al circular una corriente alterna a través de la bobina. Para lograr la circulación de la corriente adecuada, se utiliza una fuente de potencia eléctrica, así como también un generador de frecuencia controlado que, junto con las propiedades del material que conforma el núcleo, determinan la temperatura y su evolución en el tiempo.

Este método también se conoce como convertidor resonante; el cual consta de un puente inversor alimentado por una fuente de tensión continua que pasa por un generador de alta frecuencia, y esto se conecta a una carga RLC, el control de la potencia de trabajo entregada depende del voltaje suministrado a la entrada del sistema o por medio de la variación de la frecuencia de resonancia.

\section{Estado del Arte}

\section{Fundamentación teórica}

El calentamiento por inducción magnética consiste en la generación de un campo magnético que induce corrientes parásitas, llamadas también corrientes de Foucault, sobre un material ferromagnético. Para la presente investigación es necesario tener en cuenta el principio teórico de este fenómeno, el cual se basa en la aplicación de varias ecuaciones de Maxwell, las leyes de Ampere, Faraday, y el efecto Joule. El efecto joule cumple un papel fundamental, porque permite aprovechar las corrientes parásitas para generar calor. En la figura 1.1 se muestra el principio de calentamiento por inducción magnética (González, 2008)

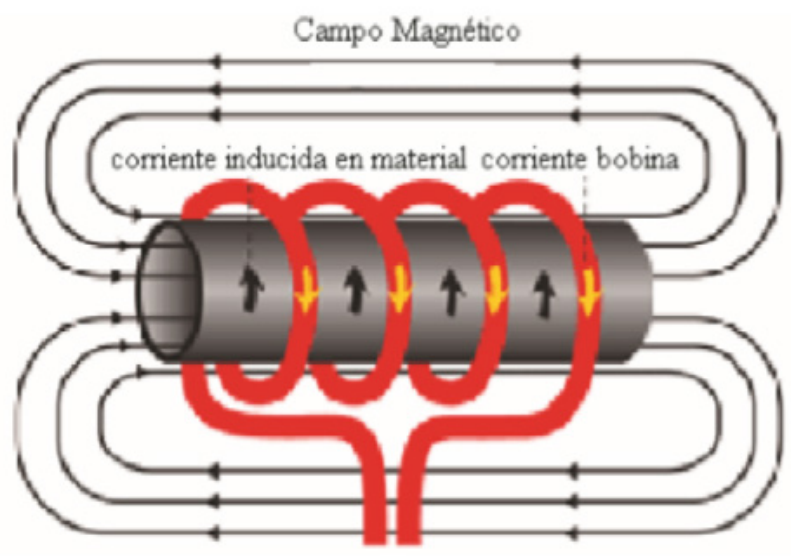


Figura 1.1 Principio de calentamiento por inducción magnética. (González, 2008) se pueden apreciar las líneas de corriente eléctrica y de flujo magnético.

Como se puede apreciar en esta figura, al aplicar una corriente variable en el tiempo a una bobina, se genera un campo magnético; y si dentro de ella se encuentra un material ferromagnético, este se calentará por inducción electromagnética. La corriente que circula por un material conductor genera un campo magnético cuya amplitud y distribución se representa matemáticamente con la ayuda de la ecuación de la ley de Ampere, como se indica en la Ecuación 1.1, donde $\mathrm{N}$ es el número de espiras del inductor; i es la corriente que lo atraviesa; $\mathrm{H}$ es la intensidad de campo magnético y $L$ es la longitud del circuito magnético.

$$
N i=\oint H . d l=H . L \quad(\text { Ecuación 1.1) }
$$

Si se aplica un voltaje variable en el tiempo, según la ecuación de Faraday, se induce un flujo magnético variable en el tiempo que se describe por la Ecuación 1.2 en donde E es el voltaje inducido; $\mathrm{N}$ es el número de espiras; $\phi$ es el flujo del campo magnético

$$
E=-N \frac{d \emptyset}{d t} \quad(\text { Ecuación 1.2) }
$$

El signo negativo de la Ecuación 1.2 indica que el voltaje inducido genera una corriente cuya dirección se opone al cambio del flujo magnético asociado, esta corriente inducida genera las corrientes de Foucault, que son responsables del calentamiento por el efecto Joule y que disipan la potencia en forma de calor (Magnetismo, 2005).

La Ecuación 1.3 permite calcular la potencia requerida para calentar el material forromagnético. En donde $\mathrm{P}$ es la potencia disipada; $\boldsymbol{i}_{\boldsymbol{f}}^{2}$ es la corriente inducida a alta frecuencia; $\boldsymbol{R}$ es la resistencia equivalente de la pieza a calentar (por donde circula la corriente inducida, debido al campo magnético variable).

\section{$P=i_{f}^{2} \cdot R_{e q} \quad(E c u a c i o ́ n ~ 1.3)$}

El funcionamiento de un calefactor por inducción electromagnética es similar al de un transformador. La bobina de inducción representa al devanado primario, mientras que el material metálico ferromagnético representa el bobinado secundario. La carga en el devanado secundario se representa por una sola espira cerrada, que simula el comportamiento de un transformador en cortocircuito. Se considera que la resistencia equivalente del secundario tiene un valor pequeño, el nivel y rapidez del calentamiento dependerá de la frecuencia de la corriente inducida y de la permeabilidad magnética del material. De acuerdo a esta ecuación, para generar pérdidas por el 
efecto Joule son necesarias grandes corrientes inducidas, debido a que la resistencia del material es muy pequeña.

\section{Pérdidas por corrientes de Foucault}

Las pérdidas de energía debido al calentamiento Joule asociadas a las corrientes parásitas de Foucault son las más importantes en materiales ferromagnéticos, tal como se dijo anteriormente. Las pérdidas por histéresis también se presentan en materiales ferromagnéticos, pero su efecto no es de mayor importancia comparado con el producido por las corrientes parásitas, por eso no se estudian en este material (Turmero P, 2018).

En los sistemas de calentamiento por inducción (CPI) el calor se produce directamente en el interior del material, por esta razón se afirma que es más eficiente que el proceso por resistencia eléctrica. Al circular una corriente variable por una bobina, el campo magnético generado permite la inducción de corrientes en otras bobinas que estén sometidas al campo magnético producido. La magnitud de las corrientes está directamente relacionada con el número de vueltas de la bobina y del material utilizado como núcleo.

El calor generado por el circuito de inducción es transmitido directamente al objeto a calentar por conducción térmica. El material inducido por las corrientes se llama susceptor ${ }^{1}$ y se construye de distintas formas geométricas según su aplicación. Los fundamentos del fenómeno de calentamiento por inducción se definen por principios fundamentales, que determinan la distribución de corriente y la energía disipada. Las ecuaciones fundamentales se muestran en la tabla 1.1.

\section{Tabla 1. 1}

Ecuaciones fundamentales

\begin{tabular}{|c|c|}
\hline Nombre & Forma integral \\
\hline $\begin{array}{l}\text { Ley de } \\
\text { Faraday }\end{array}$ & $V=-N \frac{d \emptyset}{d t}$ \\
\hline Ley de ampere & $\begin{array}{l}N i \\
=\oint H \cdot d l \\
=H . L\end{array}$ \\
\hline Ley de Joule & $P=i_{i}^{2} \cdot R_{e q}$ \\
\hline Ley de Ohm & $I=\frac{V}{R}$ \\
\hline
\end{tabular}

1. Material usado para absorber energía electromagnética

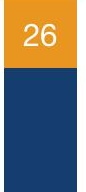




\section{Efecto pelicular}

La disminución de la densidad de la corriente desde la periferia hacia el centro del material se conoce como efecto Skin o efecto pelicular; este se presenta cuando la frecuencia del campo magnético es elevada, y se manifiesta como una disminución de las corrientes inducidas conforme se penetra en el material. Las corrientes a altas frecuencias tienden a concentrarse en la superficie del material conductor. La medida del efecto piel es la profundidad de penetración $\delta$. El efecto de difusión del campo magnético es la condición que rige al calentamiento por inducción. En una primera aproximación para un cilindro se puede asumir que la distribución del campo magnético se puede representar por la Ecuación 1.4, donde $\boldsymbol{H}(x)$ representa la coordenada radial del cilindro; $\mathrm{x}$ es la distancia; $\boldsymbol{H}_{0}$ es la intensidad del campo en la superficie; $\boldsymbol{\delta}$ es la profundidad de penetración. Se debe notar que en suma el exponente es adimensional.

$$
H(x)=H_{0} * e^{-x / \delta} \quad \text { (Ecuación 1.4) }
$$

\section{Frecuencia de Trabajo Resonante}

La frecuencia de trabajo de un sistema de calentamiento por inducción depende de la pieza de trabajo que se desea calentar y el material del que está hecha. Es importante utilizar un sistema de inducción que aplique la energía a lo largo de todo el intervalo de frecuencias adecuado para la aplicación. Para comprender mejor los motivos de estas frecuencias de trabajo diferentes, se examinará más a fondo el efecto pedicular. Cuando el campo electromagnético induce una corriente en la pieza, la corriente fluye principalmente por la superficie de la pieza. La profundidad de penetración depende de la frecuencia de trabajo, las propiedades del material y la temperatura de la pieza. Por regla general, el calentamiento de piezas más pequeñas por inducción requiere frecuencias de trabajo mayores (muchas veces por encima de los $50 \mathrm{kHz}$ ). Con las fuentes de potencia de inducción de estado sólido modernas, dotadas de sistemas de control con microprocesadores integrados, es posible conseguir fácilmente unos procesos de calentamiento repetibles y eficientes, siempre y cuando todas las piezas se sitúen en una posición uniforme dentro de la bobina (Kolocsar, 2017).

\section{La profundidad de penetración}

La medida cuantitativa del efecto piel es la profundidad de penetración del calentamiento $\delta$ y se define como aquella distancia de la superficie en la cual el valor de la corriente se ha reducido a $1 /$ e o $37 \%$ del valor que se tiene en la superficie del cilindro. La densidad de potencia en este punto es $1 / \mathrm{e}^{\wedge} 2$ o del $14 \%$ del valor de la superficie; por lo tanto, el $86 \%$ del calentamiento ocurre en el volumen comprendido entre la superficie

Dado un material con resistividad $\boldsymbol{\rho}$, permeabilidad relativa $\mu \mathrm{r}$, la permeabilidad magnética del vacío (4.ח.10ex-7) es $\boldsymbol{\mu}_{0}$, sobre el cual se aplica una onda sinusoidal con frecuencia $\boldsymbol{f}$, la profundidad de penetración de la onda seria $\delta$ que está dado por la Ecuación 1.5. 


$$
\delta=503 \sqrt{\frac{\rho}{f . \mu_{r}}} \quad \text { (Ecuación 1.5) }
$$

Se define $\delta$ como la distancia donde el campo magnético se atenúa y es la zona donde se concentra el $63 \%$ de la corriente porque es el lugar en donde la intensidad del campo disminuye a $1 / \mathrm{e}$ y el $87 \%$ de la potencia que se disipa. En la figura.1.2 se muestra la distribución de la densidad de corriente y la densidad de potencia desde la superficie hasta el interior del material.

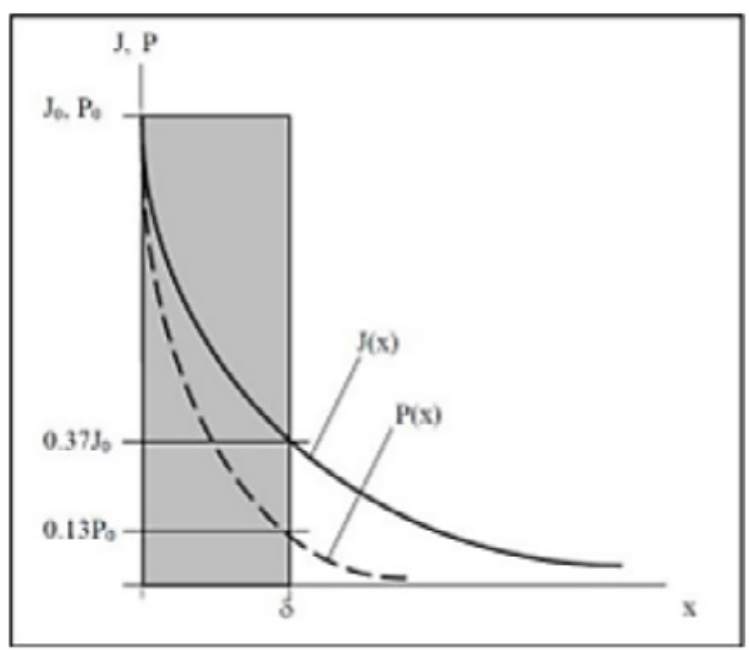

Figura 1.2. Distribución de la corriente en función de la profundidad.

Cuando la frecuencia aumenta, la profundidad de penetración disminuye (Bermúdez, 2003). Además, la profundidad de penetración estándar es inversamente proporcional a la permeabilidad magnética relativa y a la conductividad del material.

En la figura 1.3 se muestra la curva de profundidad de penetración de algunos elementos en función de la frecuencia de la onda de la fuente de alimentación.

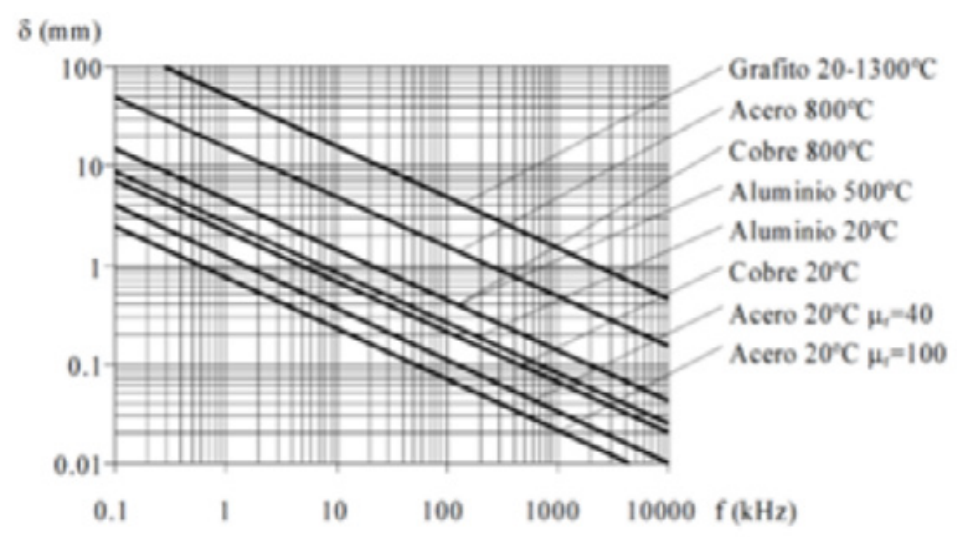

Figura 1.3. Curva de la profundidad de penetración de corrientes inducidas en función de la frecuencia. 


\section{Energía transferida en forma de calor}

Para obtener la potencia requerida por el sistema es necesario realizar el cálculo de la energía transferida en forma de calor hacia el objeto a calentar, para de esta forma realizar el correcto dimensionamiento de la fuente de alimentación. El calor es una forma de energía que es transmitida entre dos cuerpos o sistemas, y está causado por un cambio de temperatura. La diferencia de temperatura de cada sistema dependerá, entre otros, del calor específico del elemento que forma el cuerpo.

"El calor específico se define como la cantidad de calor $\boldsymbol{d Q}$ necesaria para que una unidad de $\mathrm{m}$ masa de sustancia incremente su temperatura en un grado" y lo representamos con la Ecuación 1.6 (Formula de calor específico).

$$
C=\frac{1}{m} \frac{d Q}{d T} \quad \text { Ecuación } 1.6
$$

Para variaciones no muy grandes en la temperatura del cuerpo, el valor de c es considerado como constante, entonces el valor de la energía transferida en forma de calor se expresa con la siguiente Ecuación 1.7 (Energía transferida en forma de calor):

\section{$\boldsymbol{Q}=\boldsymbol{m} \boldsymbol{c} \Delta \boldsymbol{T} \quad$ Ecuación 1.7}

Donde $Q$ es la cantidad de energía calorífica [kcal]; m la masa del objeto a calentar [kg]; c el calor específico del objeto a calentar $\left[\mathrm{kCal} / \mathrm{kg}^{\circ} \mathrm{C}\right]$, y $\Delta \mathrm{T}$ el Diferencial de temperatura del objeto $\left[{ }^{\circ} \mathrm{C}\right] . \mathrm{En}$ la tabla 1.2 podemos ver la densidad de los materiales, así como también su calor específico.

Tabla 1. 2.

Densidad y calor específico de materiales de interés

\begin{tabular}{|l|c|c|}
\hline \multicolumn{1}{|c|}{ Material } & Densidad $\left(\mathrm{Kg} / \mathrm{m}^{3}\right)$ & $\begin{array}{c}\text { Calor especifico } \\
\left(\mathrm{Kcal} / \mathrm{Kg}^{\circ} \mathrm{C}\right)\end{array}$ \\
\hline Hierro & 7.860 & 0.107 \\
\hline Acero inoxidable & 8238 & 0.11 \\
\hline Polipropileno & 946 & 0.48 \\
\hline Tungsteno & 19250 & 0.037 \\
\hline
\end{tabular}


Al aumentar la temperatura, se incrementa la resistividad por encima de la temperatura de Curie $\left(750^{\circ} \mathrm{C}\right.$ en los aceros); en tal caso el material deja de ser ferromagnético y pasa a ser paramagnético (Egas, 2016). Debido a este principio se puede decir que el inductor baja su rendimiento en el caso de que no se consiga una geometría pieza-inductor aceptable. Para el prototipo del calentador de agua de inducción electromagnética el realizar el diseño y manufactura de la bobina es de gran importancia ya que conseguir un elemento que se adapte a la aplicación resulta de un proceso de investigación y de constantes pruebas de ensayo y error.

\section{Circuito equivalente del calentador por inducción}

Si se parte del circuito equivalente básico de un transformador y se considera que el acoplamiento magnético es eficiente, se puede idealizar el circuito equivalente del calentador por inducción mediante dos inductancias acopladas, con una resistencia en paralelo al circuito secundario del transformador. En la figura 1.4 se muestra el esquema eléctrico general del sistema de inducción. Este circuito equivalente es similar al de un motor de inducción asincrónico. En la figura $1.4 \mathrm{Lp}$ es la inductancia del circuito primario; Ls es la inductancia del circuito secundario; Rs es la resistencia de dispersión que forma la espira que aparece en la carga por efecto piel, y M representa la inductancia mutua entre ambas bobinas. Lt, Rp y C corresponden a la línea de transmisión de la energía hacia la bobina de inducción (González, 2008).

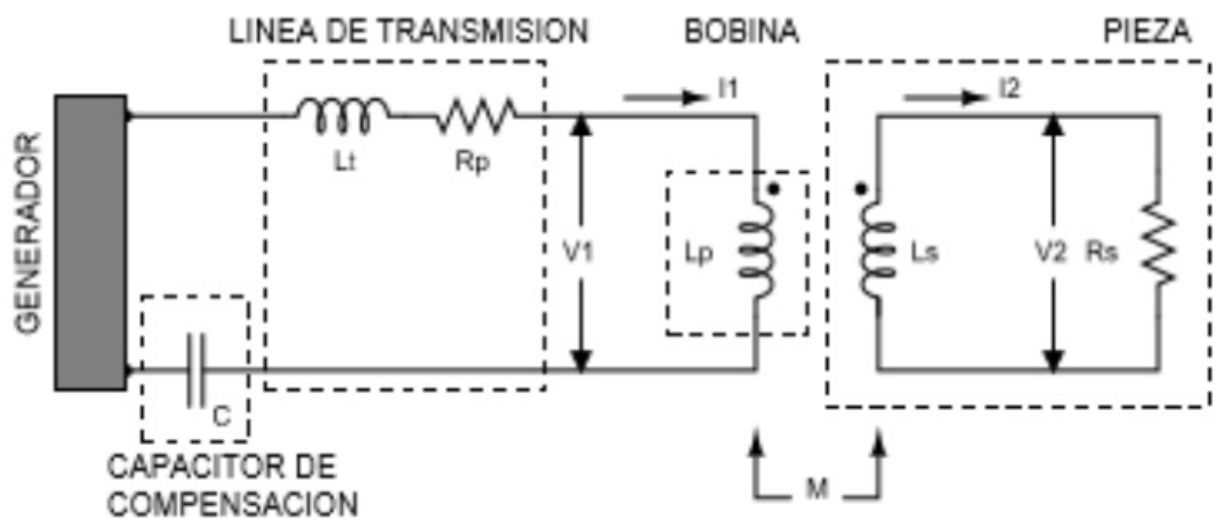

Figura 1.4. Modelo eléctrico del conjunto bobina-pieza equivalente a un transformador.

En el equipo de prueba de la presente investigación el circuito electrónico tiene un condensador en paralelo con la bobina primaria. Este condensador configura un circuito resonante en paralelo que se lo ha utilizado para garantizar la máxima transferencia de potencia entre la fuente de poder y el conjunto bobina-pieza. Para el efecto, es necesario que el sistema LC trabaje permanentemente en régimen de resonancia.

\section{Magnetización}

La magnetización es un fenómeno físico que se manifiesta en ciertos materiales ferromagnéticos ante la presencia de un campo magnético. Estos materiales muestran un codo de saturación magnética, y están compuestos de regiones microscópicas llamadas dominios magnéticos que 
actúan como pequeños imanes permanentes. Antes de que un campo magnético externo sea aplicado al material, los dominios se encuentran orientados al azar. Sus pequeños campos magnéticos apuntan en direcciones aleatorias y se cancelan entre sí, de modo que el material no produce un campo magnético global neto. Cuando se aplica un campo de magnetización $\mathrm{H}$ externo al material, lo penetra y causa la alineación de los dominios, provocando que sus pequeños campos magnéticos roten y se alineen paralelamente al campo externo, estos momentos magnéticos se suman para crear un gran campo magnético que se extiende hacia fuera del material.

Cuando prácticamente todos los dominios se encuentran alineados, ocurre el efecto de saturación por lo que cualquier incremento posterior en el campo aplicado no puede causar una mayor alineación. (Valbuena, 2003), en la figura 1.5 se indica el codo de saturación de diferentes materiales ferromagnéticos.

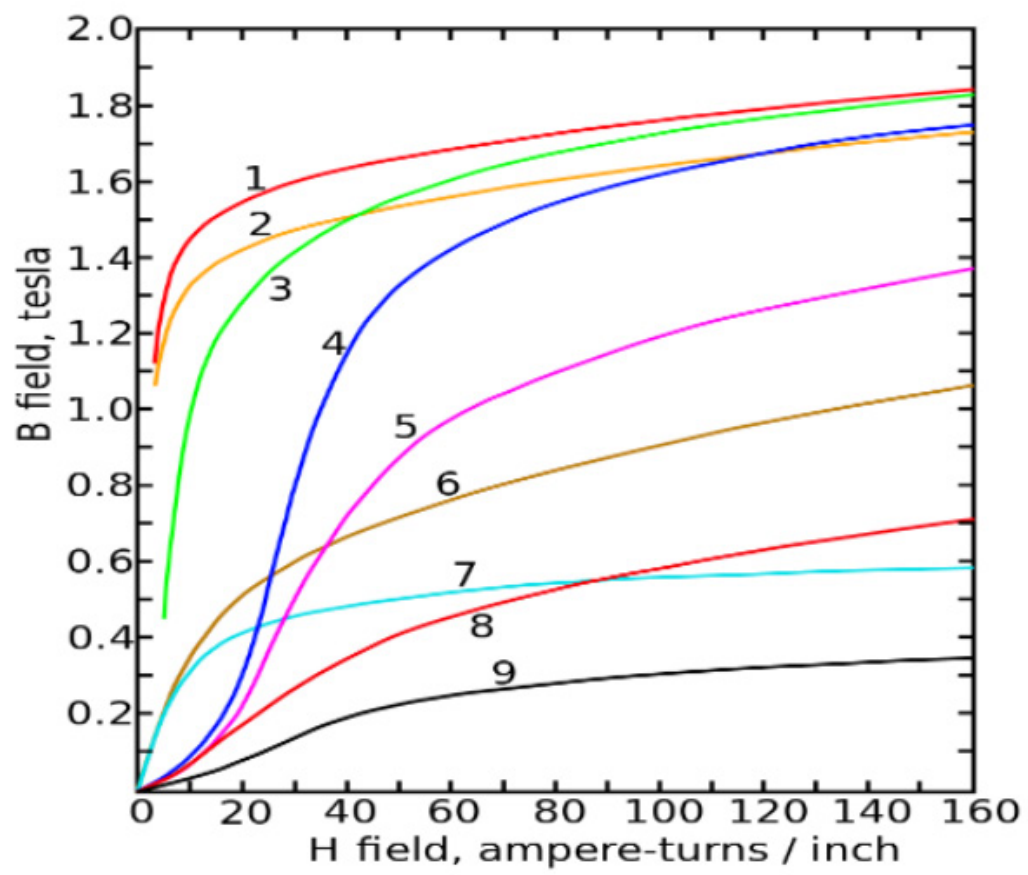

Figura 1.5. Curvas de magnetización de nueve materiales ferromagnéticos diferentes, mostrando el efecto de saturación. 1.Hoja de acero, 2.Acero al silicio, 3.Acero de crisol, 4.Acero al tungsteno, 5.Acero magnético, 6. Hierro de crisol, 7.Níquel, 8.Cobalto, 9.Magnetita.

\section{Cálculos del Circuito de Potencia.}

\section{Alimentación requerida DC para el prototipo}

Para el diseño del rectificador se considera la potencia máxima de trabajo y el voltaje de salida después del transformador y la energía rectificada. El calentador se diseñó para una potencia de $180 \mathrm{~W}$ y un voltaje de $12 \mathrm{~V} \mathrm{dc}$; con estos parámetros se determinó la corriente nominal de trabajo 
del calentador de agua. Utilizando la ecuación general se obtiene:

$$
\begin{gathered}
P=V * I \\
I_{\text {in }}=\frac{180}{12} \\
I_{\text {in }}=15 \mathrm{~A}
\end{gathered}
$$

\section{Alimentación requerida AC para el prototipo}

Para el diseño del rectificador se considera la potencia máxima de trabajo y el voltaje de entrada de la red eléctrica. El calentador se diseñó para una potencia de $180 \mathrm{~W}$ y un voltaje de $12 \mathrm{~V}$ dc; con estos parámetros se determinó la corriente nominal de trabajo del calentador de agua. Utilizando la ecuación general se obtiene:

$$
\begin{gathered}
P=V * I \\
I_{\text {in }}=\frac{180}{120} \\
I_{\text {in }}=1,5 \mathrm{~A}
\end{gathered}
$$

\section{Diseño del rectificador}

Se dimensiona el rectificador en base al voltaje pico sobre el puente rectificador y la corriente que circula por el mismo.

$$
\begin{gathered}
V_{\text {pico inver }}=2 * 12 * \sqrt{2} \\
V_{\text {pico inver }}=33,9 \mathrm{~V}
\end{gathered}
$$

Se seleccionó un puente rectificador comercial de valor estándar de 400 voltios y 35 Amperios.

\section{Diseño de la red LC}

El propósito del filtro es absorber las corrientes armónicas generadas por las conmutaciones en el inversor cuasi resonante, y evitar el cortocircuito. También ayuda a mejorar el factor de potencia del circuito, debido a que este capacitor sirve como filtro en el tanque de resonancia previniendo que circulen corrientes de alta frecuencia del inversor hacia la red eléctrica. En la referencia (Martínez, 2013) se tiene un cálculo detallado del circuito resonante RLC. En este caso la R 
representa la resistencia equivalente obtenida anteriormente.

$$
f_{c}=\frac{1}{2 \pi \sqrt{L * C}}
$$

Se asume el valor del capacitor $C_{002}=0.68 \mathrm{uF}$

Despejando L de la ecuación se obtiene

$$
\begin{gathered}
L=\frac{1}{C\left(f_{c} * 2 \pi\right)^{2}} \\
L=\frac{1}{0.68 * 10^{-6}\left(300 * 10^{3} * 2 \pi\right)^{2}} \\
L_{001}=0.4 \mu H
\end{gathered}
$$

Nuestro diseño se orienta a la construcción de un calentador de agua por inducción magnética a una frecuencia resonante de $300 \mathrm{kHz}$ (Ver anexo), así garantizamos eliminar ruidos que pueden ser perturbadores para los usuarios mientras esté encendido el calentador de agua.

\section{Estimación de la potencia necesaria para el calentador de agua}

La potencia necesaria para calentar la pieza de trabajo a una temperatura adecuada está definida por la Ecuación 1.8: Donde $\mathrm{M}$ es la masa de la pieza en $\mathrm{kg}$; c es el calor específico en $\mathrm{J} /\left(\mathrm{kg}{ }^{\circ} \mathrm{C}\right)$; T_(in ) y $\mathrm{T}_{\text {f }}$ es la temperatura inicial y final en ${ }^{\circ} \mathrm{C}$; y t es el tiempo requerido en segundos.

$$
P_{w}=M c \frac{T_{f}-T_{i n}}{t}
$$

La masa se puede determinar a través de las tablas de estandarización de tubos conforme a las normas DIN 2448 y DIN 2458, así como también a la norma europea EN 10255 de medidas y masas lineales para tubos en base a la ISO 4200 (SIKLA, 2013).

$$
\begin{gathered}
P_{w}=20 \mathrm{~kg} * \frac{239 \mathrm{~J}}{\mathrm{~kg}^{\circ} \mathrm{C}} * \frac{55-15}{1200 \mathrm{~s}}{ }^{\circ} \mathrm{C} \\
P_{w}=160 \mathrm{~W}
\end{gathered}
$$

El conjunto formado por el tubo por el cual circula el agua se convierte en un intercambiador de calor altamente eficiente. Considerando que $\mathrm{P} \_w$ representa la potencia necesaria para calentar el agua, la potencia de la fuente tiene que ser un poco mayor debido a las pérdidas de energía en los diferentes subsistemas, de esta manera la potencia efectiva que la fuente debe ser capaz de suministrar; así tenemos la Ecuación 1.9 donde $\eta \_T=\eta$ térmica * $\eta$ Eléctrica. 


$$
\begin{gathered}
P_{e}=\frac{P_{w}}{\eta_{T}} \\
P_{e}=\frac{160 \mathrm{~W}}{0.86} \\
P_{e}=186 \mathrm{~W}
\end{gathered}
$$

\section{Profundidad de penetración}

Cálculo de la profundidad de penetración que es la resultante del efecto piel. Este dato es muy importante ya que es la medida que penetra en la pieza de trabajo para obtener el calentamiento en el material ferromagnético; modificar la profundidad de penetración es posible únicamente variando la frecuencia de operación, así tenemos:

$$
\begin{gathered}
\delta=503 \sqrt{\frac{\rho}{f \cdot \mu_{r}}} \\
\delta=503 \sqrt{\frac{8.9 * 10^{-8}}{\left(3 * 10^{5}\right) *(109)}} \\
\delta=26 \mu m
\end{gathered}
$$

\section{Cálculo del número de espiras}

Para el cálculo de la bobina se debe primero considerar el flujo magnético que recorre la superficie del Fe, así como también el flujo magnético que se encuentra en el aire dentro de la bobina, así tenemos la Ecuación 1.10.

$$
\emptyset_{\text {Total }}=\emptyset_{\mathrm{Fe}}+\emptyset_{\text {aire }} \quad \text { Ecuación } 1.10
$$
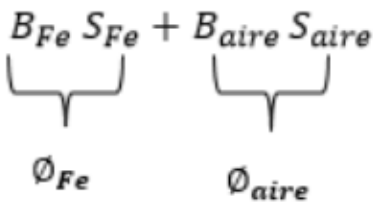

donde:

$$
S_{F e}=2 \pi R \delta ; \quad S_{\text {aire }}=\pi R^{2}
$$

$$
B_{F e}=\mu_{r} \mu_{0} H ; \quad B_{\text {aire }}=\mu_{0} H
$$

$$
\text { Flujo magnético: } \quad \emptyset_{\text {Total }}=\mu_{r} \mu_{0} H S_{F e}+\mu_{0} H S_{\text {aire }}
$$


Si se toma el factor común, se obtiene el flujo total al interior de la bobina, que está en función de la superficie transversal interior de la bobina (S_Total)

$$
\emptyset_{\text {Total }}=\mu_{0} H\left(\mu_{r} S_{F e}+S_{\text {aire }}\right)=\mu_{0} H S_{\text {Total }}
$$

Se obtiene entonces la superficie equivalente total que permite el flujo magnético. Se ha hecho este procedimiento dado que el camino magnético del flujo no atraviesa únicamente el hierro sino, aunque en mucha menor proporción, también el aire.

$$
\begin{aligned}
& S_{\text {Total }}=S_{F e}+\frac{S_{\text {aire }}}{\mu_{r}}=2 \pi R \delta+\frac{\pi R^{2}}{\mu_{r}} \\
& V_{s}=4.44 N * F * B_{\max } * S
\end{aligned}
$$

Reemplazando:

$$
V_{s}=4.44 N * F * B_{\max } *\left[2 \pi R \delta+\frac{\pi R^{2}}{\mu_{r}}\right]
$$

Despejando:

$$
\begin{aligned}
& N=\frac{V_{s}}{\left.4.44 F * \delta * B_{\max } \mid 2 \pi R \delta+\frac{\pi R^{2}}{\mu_{r}}\right]} \\
& N=\frac{15}{4.44+\left(3 * 10^{5}\right)+0.1\left[2 \pi *\left(15 * 10^{-3}\right) *\left(26 * 10^{-6}\right)+\pi \frac{\left(15^{\prime}+10^{-3}\right)^{2}}{109}\right]} \\
& N=13 \text { espiras }
\end{aligned}
$$

\section{Inversor}

Un inversor de voltaje es un dispositivo electrónico que convierte un determinado voltaje de entrada de Corriente Continua (CC) en otro voltaje de salida de Corriente Alterna (CA). Es decir, recibe corriente continua de un determinado voltaje y proporciona corriente alterna, generalmente de un voltaje diferente al de entrada. Para el presente proyecto es necesaria la implementación de una fuente de alimentación de alta frecuencia encargada de suministrar energía a la carga resonante.

\section{Inversor de medio puente}

El inversor de medio puente consta de dos interruptores, donde la tensión aplicada a la carga se divide a la mitad mediante un divisor capacitivo, este inversor puede generar una salida con forma de onda cuadrada o una salida bipolar con modulación por ancho de pulsos. 

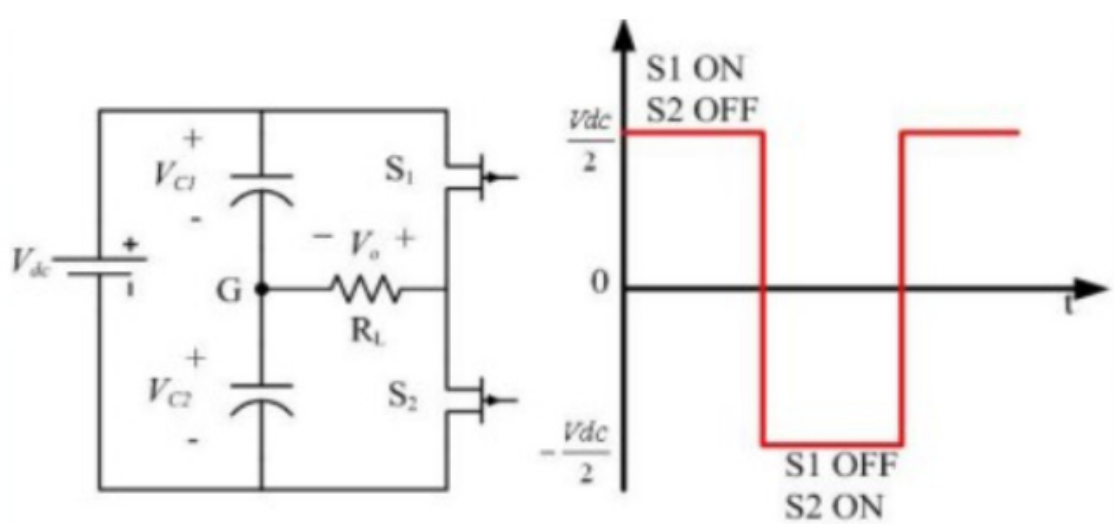

Figura 1.6. Inversor de medio puente.

\section{Secuencia de conmutación}

Sw1 cerrado, Sw2 abierto y el voltaje del capacitor C1 se ve reflejado en la carga; mientras que al estar Sw2 cerrado, Sw1 abierto y el voltaje de entrada Vs/2 aparece a través de la carga. El voltaje de salida en la carga corresponderá a una señal cuadrada.

Considerando que los capacitores C1 y C2 están cargados a la mitad del voltaje Vs, tal como se observa en los capacitores flotantes de la figura 1.6. Para la forma de onda del inversor de medio puente en la primera mitad del periodo T/2 se obtendrá un voltaje con forma de onda cuadrada $\mathrm{Vs} / 2$. Para la segunda parte del periodo $\mathrm{T} / 2$ a $\mathrm{T}$ se polariza de forma inversa y el voltaje de salida será $-\mathrm{V} / 2$ tal como se describe en la tabla 1.3.

Tabla 1.3

Posición de los Mosfet

\begin{tabular}{|l|l|}
\hline Interruptores cerrados & Voltaje \\
\hline (S1) & $+\mathrm{Vs} / 2$ \\
\hline Todos abiertos & 0 Tiempos muertos \\
\hline (S2) & $-\mathrm{Vs} / 2$ \\
\hline
\end{tabular}

\section{Control de inversor de medio puente}

Existe un tiempo muerto intermedio denominado banda muerta $\mathrm{Td}$, el cual ayuda a prevenir un cortocircuito al cambiar de estado de los interruptores. 


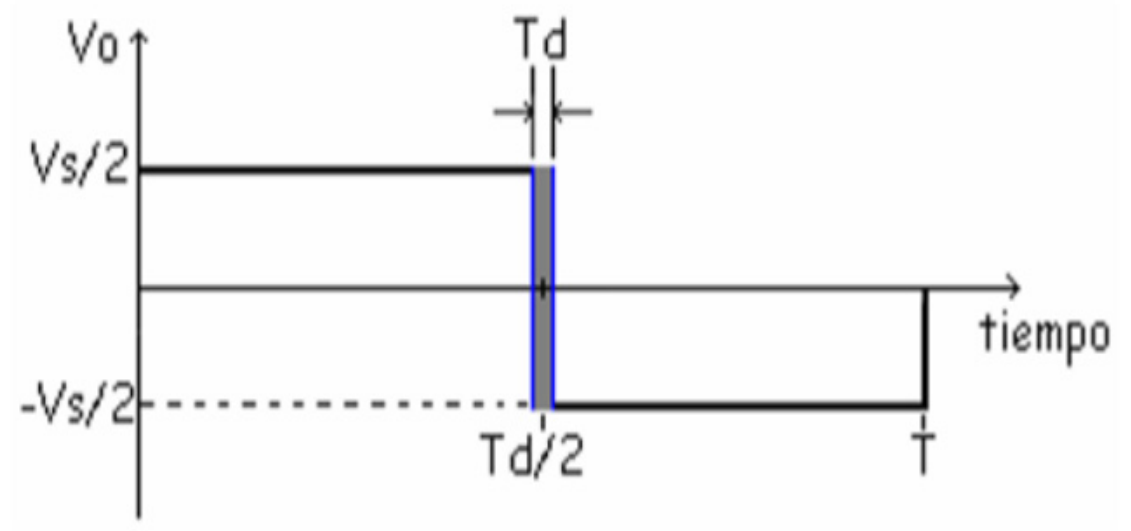

Figura 1.7. Voltaje de salida del inversor de medio puente.

\section{Circuito inversor utilizado}

El circuito inversor que se utilizó para el prototipo funciona en forma similar al inversor de medio puente ya explicado, con la diferencia de que la conmutación de los transistores Mosfet se realiza con el mismo condensador que entra en resonancia. El condensador en este caso se carga con voltaje negativo que ingresa en la compuerta del Mosfet y lo apaga momentáneamente, mientras que el otro Mosfet del medio puente queda encendido.

\section{RESULTADOS}

\section{Pruebas de funcionamiento}

Se denomina circuito LC porque tiene incorporado una bobina y un condensador para el control $y$, dependiendo del sentido de la corriente que circula por la bobina, genera un campo magnético que induce una corriente en un material ferromagnético, el cual ante dicha corriente comienza a calentarse.

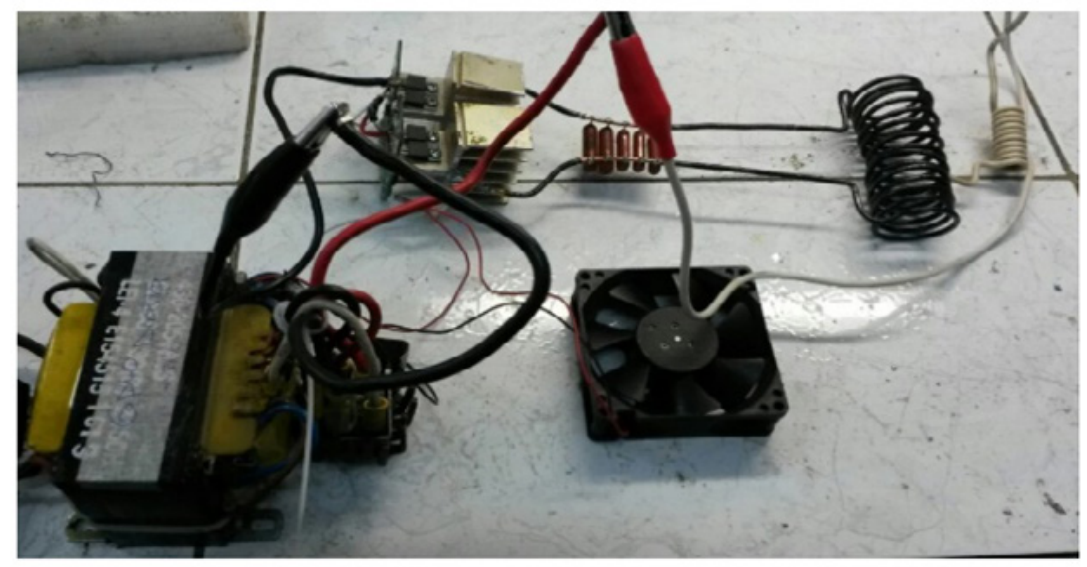

Figura 1.8. Circuito resonante. 
El sistema de calentamiento se construyó con una tubería de acero galvanizado. Se usó este material por ser el más común en las instalaciones hidrosanitarias de los hogares y por las características ferromagnéticas que le proporciona el acero que lo compone, así como por el recubrimiento de zinc, que no permite que el acero se oxide ante el paso del agua; permite comportarse para la aplicación como un núcleo ferromagnético, el cual permite generar la inducción electromagnética en conjunto con la bobina general del sistema de calentamiento figura 4.9.

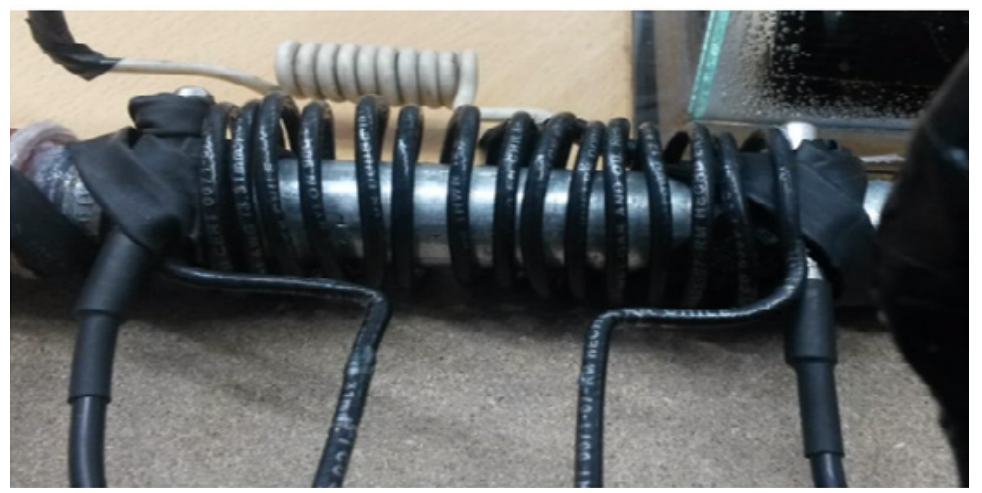

Figura 1.9. Bobina de trabajo con núcleo ferromagnético.

A continuación se presenta un inversor de medio puente construido con MOSFET, en anti-paralelo a ellos se encuentran diodos de recuperación rápida, esto se debe a que los diodos internos con los que cuentan estos transistores tienen características limitadas en tiempos de recuperación inversa, por lo tanto, para anular su funcionamiento es necesario incorporar diodos en serie con los MOSFET, luego son montados en unas placas de aluminio (como se muestra en la figura 1.10) que ayudan a disipar el calor generado por su funcionamiento a plena carga.

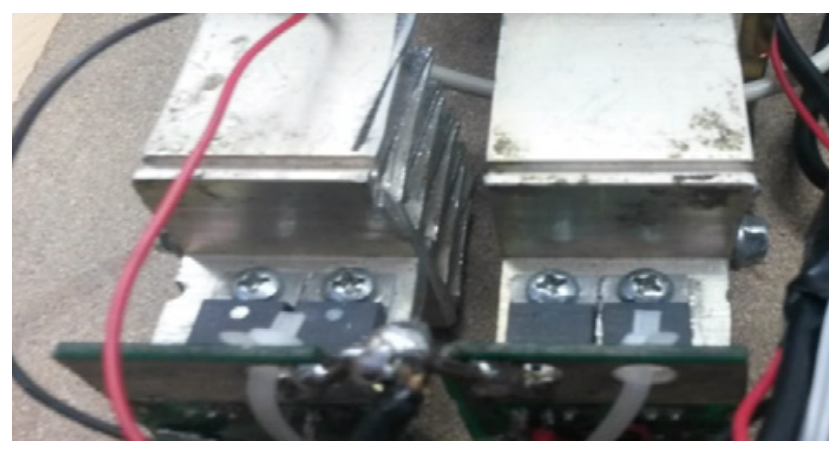

Figura 1.10. Circuito oscilador de alta frecuencia.

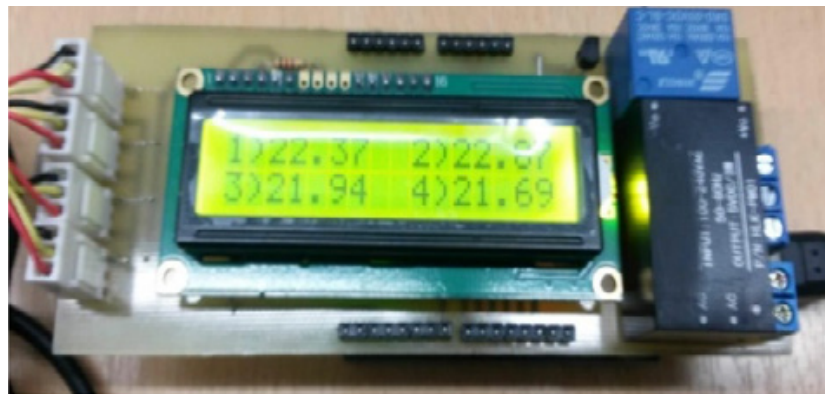

Figura 1.11. Placa electrónica para el control. 


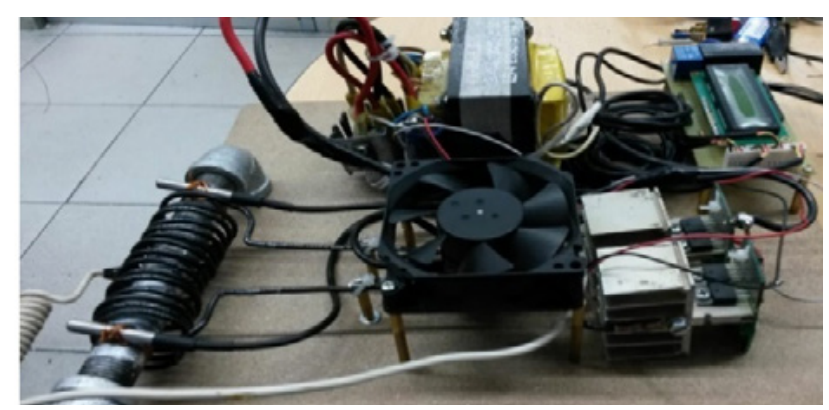

Figura 1.12. Sistema implementado del calentador de agua por inducción.x

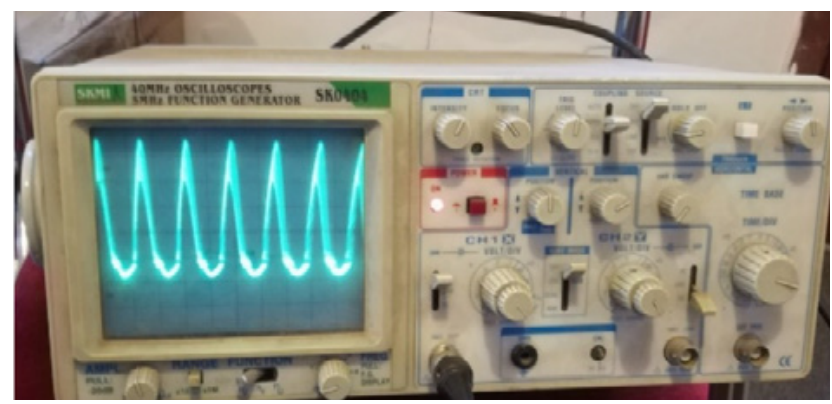

Figura 1.13. Osciloscopio SKMI (SK0404).

En el oscilograma de la figura 1.13 se puede apreciar que la onda producida en el inversor es aproximadamente sinusoidal, aunque el pico positivo es más agudo que el pico negativo. El período de cada onda medida fue de 1,7 cuadros con base de tiempos en $2 \mu$ s por división; esto es 3,4 $\mu \mathrm{s}$. De esta manera, la frecuencia de la oscilación se calculó en $288 \mathrm{kHz}$. De la misma manera, se midió la altura total del pico de la onda con una punta de prueba por 10 con un total de 5,2 cuadros en la escala de 1 Voltio por división. Esto daría un voltaje de 52 voltios pico-pico. Luego de realizar los cálculos correspondientes esto daría un voltaje de 18 Voltios RMS.

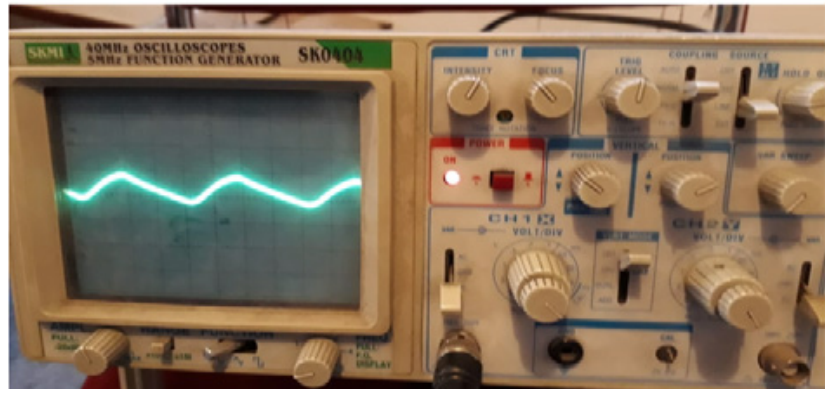

Figura 1.14. Onda rectificada y filtrada.

En el oscilograma de la figura 1.14 se puede observar la onda rectificada y filtrada que alimenta al inversor auto-oscilante. La oscilación tiene un período de $8,3 \mathrm{~ms}$; es decir, tiene una frecuencia de $120 \mathrm{~Hz}$, tal como podía esperarse de un circuito rectificador de media onda. Se ha medido esta oscilación en el modo AC para intensificar la escala. El voltaje pico de rizado medido es de 5 Voltios pico a pico. Si se requiere mayor potencia de salida del inversor, sería necesario añadir un condensador de mayor valor para el filtrado de la señal. El Voltaje DC obtenido fue de $20 \mathrm{~V}$ pico con un valle que llegaba hasta los 15 Voltios DC. 


\section{Conclusiones}

- En un sistema de calentamiento se deben tener en cuenta las propiedades magnéticas y de resistencia del material a calentar, además de la forma, para realizar un correcto diseño de la bobina de inducción. Sin embargo, no son los únicos parámetros para realizar el diseño del sistema de calentamiento por inducción, hay que tener en cuenta la temperatura que se requiere y el tiempo de calentamiento para así tener un valor aproximado de la potencia que requiere la carga para su calentamiento.

- El controlador del sistema es parte fundamental para el calentamiento por inducción, ya que si se requiere sacar la máxima eficiencia necesariamente se debe estar regulando la frecuencia de resonancia o controlando el nivel de voltaje y/o corriente en la carga, lo que conlleva implementar otro tipo de sensores en el sistema

- Existe una gran variedad de inversores resonantes para construir sistemas de calentamiento por inducción electromagnética, que depende de la aplicación, del capital que se pueda invertir en cuanto a componentes y elementos del sistema y de la complejidad que este lleve para realizar el calentamiento en la carga. Para cada caso particular se deberá utilizar la opción que más se ajuste a alguna de las anteriores condiciones. 


\section{Referencias Bibliográficas}

Álvarez, M. S. (Abril de 2009). modelación y control de un sistema piloto de calentamiento de fluidos por inducción magnética. Recuperado de http://repositorio.uchile.cl/bitstream/handle/2250/102050/sanchez_ ma.pdf?sequence $=3$

Bermúdez, M. (2003). Estudio y diseño de un calentador por inducción magnetica . Recuperado de http://lep. eie.pucv.cl/TesisLeoFinal.pdf

Egas, E. (2016). Introducción a la Teoría Electromagnetica.

González, W. (2008). Diseño de una bobina de inducción para fundicion de titanio.

González, W. (Abril de 2008). Diseño y construcción de bobina de calentamiento por inducción para fundicion de titanio.

Kolocsar. (30 de Noviembre de 2017). Calentamiento Por Induccion Magnética. Recuperado de https://kolocsar. com/calentamiento-por-induccion-magnetica/

López, C. T. (14 de Junio de 2016). electromagnetismo. Recuperado de https://culturacientifica.com/2016/06/14/ descubrimiento-la-induccion-electro-magnetica-1/

Magnetismo. (20 de Abril de 2005). lecciones de electrónica . Recuperado de http://www.ifent.org/lecciones/ cap07/cap07-08.asp

Martínez, J. A. (2013). Desarrollo de un Inversor para Calentamiento por Inducción Electgromagnética. Mexico D.F.

SIKLA. (10 de 2013). Tubos (medidas y pesos). Recuperado de https://www.sikla.es/fast/600/17\%20Tubos\%20_ medidas\%20y\%20pesos_.pdf

Turmero, P. (08 de Enero de 2018). Programa de eficiencia energética para cocción por inducción. Recuperado de http://www.monografias.com/trabajos102/cocinas-induccion-y-calentamiento-agua/cocinas-induccion-y-calentamie

Valbuena, M. (Abril de 2003). Propiedades magnetica de los materiales. Recuperado de http://www.quieroapuntes.com/propiedades-magneticas-de-los-materiales_1.html. 
